\title{
Short-Term Effect of Erythropoietin on Brain Lesions and Aquaporin-4 Expression in a Hypoxic-Ischemic Neonatal Rat Model Assessed by Magnetic Resonance Diffusion Weighted Imaging and Immunohistochemistry
}

\author{
OLIVIER BRISSAUD, FRÉDÉRIC VILLEGA, JAN PIETER KONSMAN, STÉPHANE SANCHEZ, GÉRARD RAFFARD, \\ JEAN-MICHEL FRANCONI, JEAN-FRANÇOIS CHATEIL, AND ANNE-KARINE BOUZIER-SORE
}

\begin{abstract}
Neonatal Intensive Care Unit [O.B., F.V.] and Pediatric Radiological Department [J.-F.C.], University Children's Hospital, Bordeaux 33076, France; Psychoneuroimmunology, Nutrition, and Genetics Laboratory [J.P.K.] and Magnetic Resonance of Biological Systems Center [S.S., G.R., J.-M.F., J.-F.C., A.-K.B.-S.], CNRS UMR 5226, University of Bordeaux 2, Bordeaux 33076, France
\end{abstract}

\begin{abstract}
Erythropoietin (Epo) is an endogenous cytokine that regulates hematopoiesis and is widely used to treat anemia. In addition, it has recently increased interest in the neurosciences since the new concept of Epo as a neuroprotective agent has emerged. The potential protective effect of human recombinant Epo (r-hu-Epo) on a hypoxic-ischemic (HI) pup rat model was studied. Cerebral HI was obtained by permanent left carotid artery ligature of pups followed by a 2-h hypoxia. Three hours after carotid occlusion, brain lesions were assessed by magnetic resonance diffusion weighted imaging. Intraperitoneal administration of r-hu-Epo $(30,000 \mathrm{U} / \mathrm{kg}$ dose) limited both the HI-induced brain lesion area and the decrease in apparent diffusion coefficient (ADC) in the lesion. To identify potential mechanisms underlying the effects of Epo, immunohistochemical detection of caspase-3 and water channel protein aquaporin-4 (AQP4) were performed. No early apoptosis was detected, but up-regulation of AQP4 expression was observed in HI pups that received r-hu-Epo compared with HI animals without treatment. This study demonstrates an early neuroprotective effect of Epo with regard to brain lesion area and ADC values. One possible mechanism of Epo for decreasing brain edema and cellular swelling could be a better clearance of water excess in brain tissue, a process possibly mediated by AQP4. (Pediatr Res 68: 123-127, 2010)
\end{abstract}

$\mathrm{N}$ eurologic protection in perinatal hypoxia-ischemia (HI) remains a challenge and treatment outcomes have been rather poor until now (1). Several neonatal animal models have been developed to study this disease and its associated physiopathology (2-4). MRI, including diffusion weighted imaging (DWI), is a method of choice to explore the brain because it offers a noninvasive way to probe microscopic structural information on brain in vivo (5). Fractional anisotropy (FA) and the apparent diffusion coefficient (ADC) are known to reflect local microstructural properties. DWI provides excellent contrast visualization of asphyxia-induced brain lesions, in which ADC measurements show early re-

Received February 1, 2010; accepted April 28, 2010.

Correspondence: Olivier Brissaud, M.D., Ph.D., University Children's Hospital, Place Amélie Raba-Léon, 33076 Bordeaux, France; e-mail: olivier.brissaud@chu-bordeaux.fr Supported by the CNRS. stricted water diffusion, a common feature of cerebral tissue abnormalities in relation with cytotoxic edema (6).

Over the past decade, various candidate molecules for neuroprotection have been studied in neonatal rat models, including minocycline (7), allopurinol (8), deferoxamine (9), and magnesium sulfate (10). Erythropoietin (Epo), a hemopoietic growth factor, has emerged as one of the most interesting neuroprotective agents $(11,12)$. Epo was originally used in patients suffering from anemia to stimulate red blood cell proliferation, particularly in premature babies (13). The neuroprotective properties of Epo have been known for several years, even if its underlying mechanisms remain unclear $(14,15)$. However, experiments addressing the effect of Epo on HI have mostly been performed by histologic methods and only a few used an in vivo imaging approach $(16,17)$.

The aim of our study was to evaluate the potential early neuroprotective effect of Epo with DWI performed $3 \mathrm{~h}$ after an $\mathrm{HI}$ insult in the neonate rat. We measured infarct size, FA, and $\mathrm{ADC}$ values in the lesion. We also performed immunohistochemistry to detect an early apoptosis induction with caspase- 3 antibodies and the expression of the water channel protein aquaporin-4 (AQP4), because Epo is known to modulate water permeability (18). We hypothesize that Epo may represent an early neuroprotective agent in Hypoxic-Ischemic condition in neonatal rat model of Vanucci-Rice and that effect may be mediated by an AQP4 up-regulation in injured tissues.

\section{MATERIALS AND METHODS}

The study has been approved by our Institutional Animal Care and Use Committee (agreement number 33-10-003).

Animal model. Wistar rats of postnatal day (P) 7 or 8, weighing $19.8 \pm$ $2.7 \mathrm{~g}$ (no statistical difference concerning body weight between groups) were divided into three groups: I, control $(n=5$, two P7 and three P8); II, HI (ischemia +2 h hypoxia, $n=16$, eight P7 and eight P8); III, HI-Epo (ischemia + intraperitoneal injection of Epo +2 h hypoxia, $n=17$, eight P7

Abbreviations: ADC, apparent diffusion coefficient; AQP4, aquaporin-4; DWI, diffusion weighted imaging; Epo, erythropoietin; r-hu-Epo, human recombinant Epo; Epo-R, Epo receptor; FA, fractional anisotropy; HI, hypoxia-ischemia 
and nine P8). Pups were subjected to the surgical procedure described by Rice et al. (2). Briefly, pups were anesthetized with isoflurane (4\% induction, $1.5 \%$ maintenance). After a midline neck incision under local anesthesia (bupivacaine $0.5 \%$ ), the left common carotid artery was isolated then permanently ligatured (Vicryl 7/0). After surgery (never exceeded $20 \mathrm{~min}$ ), animals were allowed to recover $(30 \mathrm{~min}$ ) on a heated mattress to maintain body temperature. Pups were then placed in a hypoxic chamber of $840 \mathrm{~cm}^{3}(15 \times 7 \times 8$ $\mathrm{cm}$ ) and exposed to hypoxia (8\% oxygen-92\% nitrogen) for $2 \mathrm{~h}$. Hypoxic level was monitored by an oximeter (Airox BioMS, Pau, France) and maintained between 7.9 and $8.1 \%$ of oxygen. Chamber temperature was maintained at $31-33^{\circ} \mathrm{C}$ and humidity $>80 \%$. After hypoxic exposure, rat pups were returned to their heated mattress until MRI, performed $30 \mathrm{~min}$ later $(3 \mathrm{~h}$ after the end of the ligature). For MRI, pups were anesthetized with isoflurane (4\% induction, $1.4 \%$ maintenance), and respiration was monitored by a ventral pressure sensor. Warmed water circulating in the gradient coil system was used to maintain body temperature. Fifteen pups were killed by decapitation immediately after MRI (group I, $n=4$; group II, $n=6$; and group III, $n=5$ ). Brains were rapidly removed, rinsed in PBS, and immersion fixed in $4 \%$ paraformaldehyde in PBS (over night). Before being frozen, fixed brains were rinsed in PBS glucose (10\%).

Drug and dose. Epo (r-hu-Epo, Eprex, 4000 UI/0.4 mL, Janssen-Cilag, Issy-les-Moulineaux; France) was administered intraperitoneally immediately after carotid ligature and before hypoxia at a dose of 30,000 UI/kg [dose known to achieve the most successful neuroprotective response (19)]. Epo administration was not randomized or blinded.

MRI. Each pup was studied $3 \mathrm{~h}$ after carotid ligature on a horizontal $4.7 \mathrm{~T}$ Biospec 47/50 system (Bruker, Ettlingen, Germany) equipped with a 6-cm BG6 gradient system capable of $950 \mathrm{mT} / \mathrm{m}$ maximum strength. Measurements were performed with a birdcage resonator $(35-\mathrm{mm}$ diameter and $80-\mathrm{mm}$ length) tuned to $200.3 \mathrm{MHz}$. Coronal T2-weighted images of the brain were obtained using a RARE sequence, three slices, 2 -mm thick, FOV $2.5 \times 2.5$ $\mathrm{cm}$, TE $100 \mathrm{~ms}$, TR $5000 \mathrm{~ms}$, rare factor 8 , matrix $128 \times 128$. DWI was performed with a pulsed gradient spin echo sequence derived from the Stejskal-Tanner relationship using the following parameters: one coronal slice, localized at the level of the caudate nucleus, 2-mm thick, FOV $2.5 \times 2.5$ $\mathrm{cm}$, TE $20 \mathrm{~ms}$, TR $1800 \mathrm{~ms}$, matrix $128 \times 128, \delta 5 \mathrm{~ms}, \Delta 8.598 \mathrm{~ms}, 12$ directions, $b$ values $0-400-1000 \mathrm{~s} / \mathrm{mm}^{2}$. Duration of DWI acquisition was 96 min.

Data processing. DW images and ADC cartographies were postprocessed by using CCHIPS software under Linux. Stroke area was outlined manually on the ADC maps then measured by the software as a number of pixels. Mean values of $\mathrm{ADC}\left(\mathrm{mm}^{2} / \mathrm{s}\right)$ and $\mathrm{FA}$ in the selected area were obtained simultaneously.

Immunohistochemistry. Twenty micrometer-thick coronal brain slices were cut on a cryostat $(-1 \mathrm{~mm}$ to $+1 \mathrm{~mm} /$ Bregma) Briefly, sections were postfixed in $4 \%$ paraformaldehyde (PBS, $10 \mathrm{~min}, 4^{\circ} \mathrm{C}$ ), followed by two rinses (PBS, $10 \mathrm{~min}$ ) and two washes (PBS, $2 \%$ gelatin, $0.25 \%$ triton, $10 \mathrm{~min}$ ). Slices were then incubated with primary AQP4 or caspase- 3 antibody (overnight, room temperature, 1:500 in PBS $2 \%$ donkey serum, Sigma Chemical Co--Aldrich, Germany). After two rinses (same buffer), sections were treated for 30 min with $0.3 \% \mathrm{H}_{2} \mathrm{O}_{2}$ and again washed in PBS. Sections were subsequently incubated with biotinylated donkey anti-rabbit immunoglobulins (90 min, diluted 1:200, Jackson Immunoresearch Laboratories, Inc.) and with a streptavidin-biotin-peroxidase complex (90 min, diluted 1:1000, Vector Laboratories, Burlingame, CA). Afterward, slices were immersed in Tris-buffered saline $(0.05 \mathrm{M}, 10 \mathrm{~min})$ and in sodium acetate $(0.1 \mathrm{M} ; \mathrm{pH}=6$, $10 \mathrm{~min})$. Sections were then stained using diaminobenzidine. Finally, sections were air-dried, dehydrated in graded ethanol solutions, defatted in xylene and coverslipped in Depex mounting medium (VMR International Ltd, Poole, England). Stained sections were examined through a $10 \times$ objective mounted on a transmitted light microscope and images were captured using a high resolution CCD video camera (Nikon DMX1200 and Eclipse E400, Champigny sur Marne, France). Image processing was performed using a National Institutes of Health imaging software, Scion Image (Scion, Frederick, MD) on gray images by defining brightness and surface above which labeling had to be taken into account. Once established, these parameters remained unchanged. The image was then converted to a binary image, and the caspase-3 and AQP4 immunostaining areas were measured specifically in the somatosensory cortex.

Statistical analysis. Results were expressed as means $\pm \mathrm{SD}$ or median as appropriate. For imaging analysis, comparison of stroke area, ADC, and FA values between groups II and III were performed by a $t$ test. Inter and intraobserver error concerning stroke area, FA, and ADC value measurements were expressed by calculating the coefficient of variability $(\mathrm{CV}=\mathrm{SD} /$ mean) after repeated measurements. Intraobserver variability was calculated after three measurements each separated by 1 mo for the first 10 rats. Interobserver variability was also calculated after three measurements done by two observ-

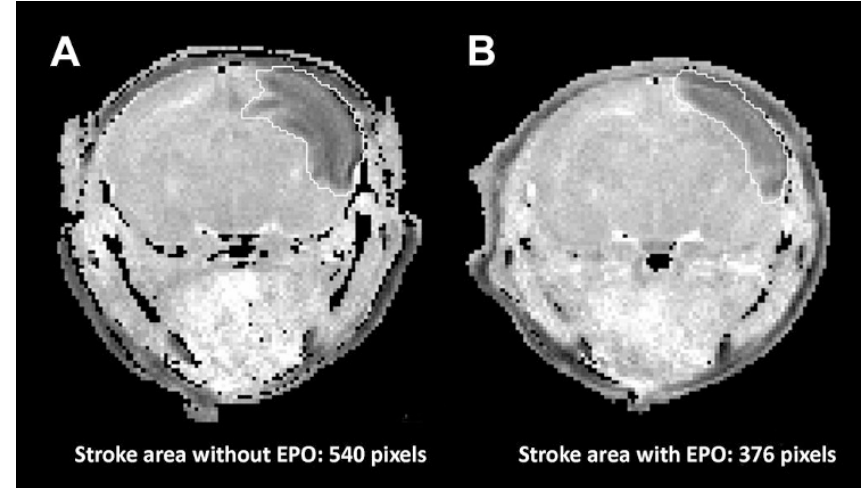

Figure 1. Example of $\mathrm{HI}$ injury area (in number of pixels) between a group II $(A)$ and a group III $(B)$ pup. Statistical difference was observed between group II (639 \pm 349 pixels; $n=16)$ and group III (394 \pm 271 pixels, $n=17)$, $p=0.006$

ers at three different times for the first 10 rats. No statistical difference was found within and between observers.

Immunostaining area was expressed as percentage of total image surface. Mann-Whitney statistical test was used to compare immunostaining areas between groups. The $p<0.05$ was considered significant.

\section{RESULTS}

Epo effect. HI injury appeared as an easily identifiable hypointensity of signal area in comparison with the whole brain, allowing its limits to be plotted by a computer mouse (Fig. 1). HI injury areas, ADC, and FA values in the lesion were measured for each rat and are presented as mean \pm SD in Fig. 2. The mean HI injury area values in groups II and III were $639 \pm 349$ pixels and $394 \pm 271$ pixels, respectively $(p=0.03)$, indicating a lesion size in HI pups treated with Epo reduced by $40 \%$ in comparison with nontreated rats (Fig. 1).

The mean FA values in HI injury area did not differ between groups II and III $(0.305 \pm 0.065$ and $0.304 \pm 0.06$, respectively; $p=0.95$ ).

The mean values of $\mathrm{ADC}$ in $\mathrm{HI}$ lesion were $0.565 \times 10^{-3} \pm$ $0.086 \times 10^{-3} \mathrm{~mm}^{2} / \mathrm{s}$ and $0.695 \times 10^{-3} \pm 0.15 \times 10^{-3}$ $\mathrm{mm}^{2} / \mathrm{s}$, respectively, in groups II and III $(p=0.006)$. Compared with ADC values found in controls $\left(0.99 \times 10^{-3} \pm\right.$ $\left.0.07 \times 10^{-3} \mathrm{~mm}^{2} / \mathrm{s}\right)$, a $45 \%$ decrease was observed in group II whereas only a $30 \%$ decrease was found in group III.

Immunohistochemistry. In control brains, no caspase-3 immunostaining was observed, whereas only a few caspase3 -positive cell bodies were found in groups II and III.

In control animals, we observed only a weak AQP4 immunostaining in the choroid plexus, and no significant labeling in the somatosensory cortex. In groups II and III (Fig. 3), positive AQP4 immunostaining was observed around small cerebral vessels located in both the white and gray matter. The intensity of AQP4 immunostaining in the injured primary somatomotor area showed significant increases in group III compared with group II [median $4.5 \%(1.1-8.8)$ versus $0.5 \%$ $(0.2-1.4), p=0.01]$. In contrast, no difference in AQP4 immunostaining in the healthy contralateral cortex was found between groups II and III. In group III, there was a significant ipsi/contralateral hemispheric difference in AQP4 immunoreactivity with increased immunostaining in the injured cortex 

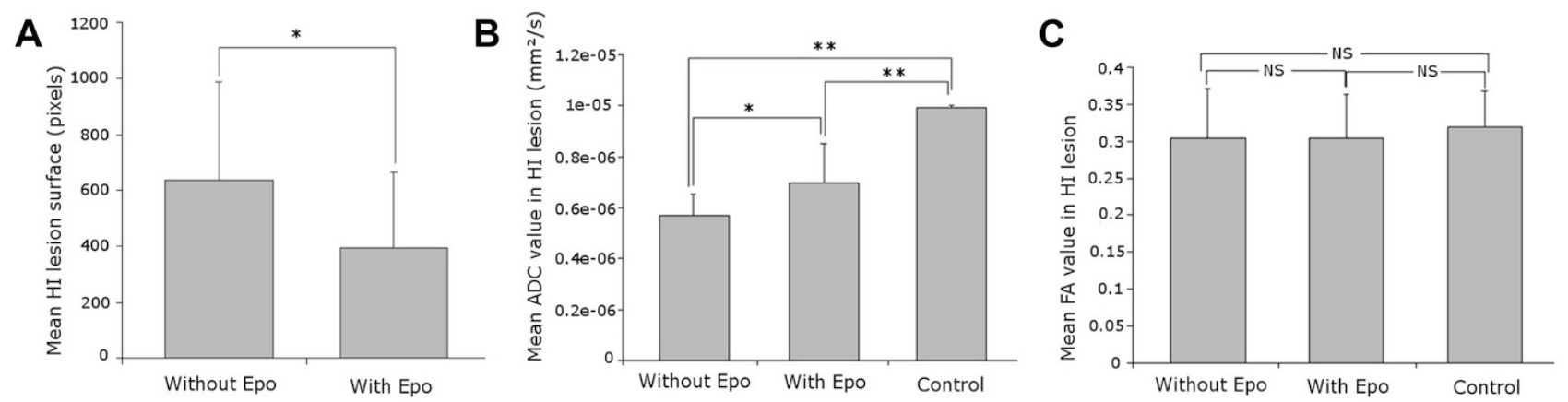

Figure 2. Comparison between mean HI injury area $(A)$, mean $\mathrm{ADC}(B)$, and mean FA $(C)$ values in HI lesion in pups not treated, treated with Epo, and controls. Statistical differences were observed regarding mean ADC values in HI lesion between group the three groups $\left(* p<0.05 ; * *<10^{-5}\right)$ but not for FA values (NS, not significant).
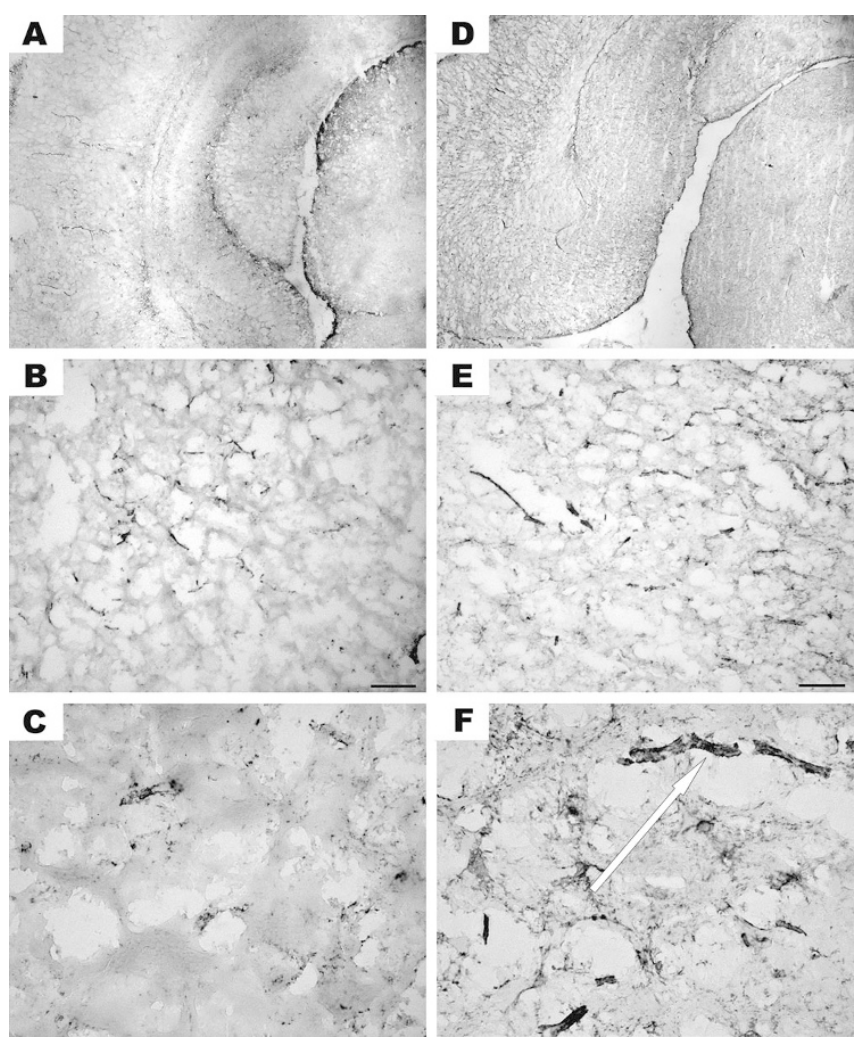

Figure 3. AQP4 immunohistochemistry. Micrographs of AQP4 immunostained sections from the left primary somatomotor cortex of a rat from group II ( $A$, magnification $\times 2 ; B, \times 10 ; C, \times 40)$ and group III $(D$, magnification $\times 2$; $E, \times 10 ; F, \times 40$ ), shown in the inset at higher magnification (scale bar 100 $\mu \mathrm{m}$ in $10 \times$ magnification) in the immunostaining observed predominantly around small cerebral vessels (white arrow on F). The intensity of AQP4 immunostaining in the injured primary somatomotor area showed significant increases in group III compared with group II (median $4.5 \%$ vs $0.5 \%, p=0.01$ ).

[medians $4.5 \%(1.1-8.8)$ versus $0.6 \%$ (0.0 and 3.7), $p=$ 0.028]. This was not observed in group II.

\section{DISCUSSION}

We showed in our study that a HI lesion size in pups treated with Epo was reduced by $40 \%$ in comparison with nontreated rats. We also observed a $45 \%$ decrease in ADC values in group II, whereas only a $30 \%$ decrease in group III (compared with ADC values measured in controls). No difference in FA values in stroke area was found between groups II and III. Immunohistochemistry results showed a link between ADC values in $\mathrm{HI}$ injuries and AQP4 expression in the brain: the highest ADC values in the HI injury area in Epo-treated rat pups corresponded to the highest levels of brain AQP4 expression.

Methodological considerations. Late DWI exploration ( $>24 \mathrm{~h}$ after brain injury) in neonatal HI rats has been reported $(20,21)$. We chose to explore neonatal HI rats shortly after carotid ligation. Early DWI explorations after neonatal rat brain injury have been also reported in the literature (22,23). In 1995, Rumpel et al. (24) used DWI and histology to explore neonatal rat brain after an $\mathrm{HI}$ insult to evaluate short- and long-term consequences. They showed that unilateral carotid ligature-induced ischemia lasting $>30$ min causes an unequivocal cytotoxic edema, followed by a more extensive vasogenic edema after $12-24 \mathrm{~h}$, preceding histologic markers of cerebral infarction. The accuracy of DWI for detecting $\mathrm{HI}$ lesion in neonatal rat brain shortly after injury was well documented in the article written by Fau et al. (23). However, to our knowledge, no publication has yet reported early DWI evaluation of the neuroprotective Epo effects in the neonatal $\mathrm{HI}$ rat model. We showed in our study that the lesion size in HI pups treated with Epo was reduced by $40 \%$ in comparison with nontreated rats. This strong difference should be slightly minimized because, as already described, variability in brain lesion size exists in this Vannucci-Rice model. This may explain some overlapped results found between stroke areas measured in groups II and III. To overcome this potential bias, we used more than fifteen pups in each group.

No difference was observed between genders with regard to Epo effect even if some striking differences were found by others (25). However, Wen et al. explored rats 6-12 wk after the HI event, thus later than in this study.

Epo administration and HI injury area. Many studies have reported histologic or immunohistochemical results after Epo administration in HI rat pups $(19,24,26-28)$ but no study have yet compared in vivo imaging in $\mathrm{HI}$ between rat pups treated or not with Epo. In adult rats, Epo injection $(5 \mathrm{U} / \mathrm{g}$ ) before and up to 3 hours after focal brain ischemia reduced injury by 60 and 55\%, respectively. In a histologic study on P7 pups, infarct volumes were reduced by 50\% after Epo injection $4 \mathrm{~h}$ before carotid ligature and hypoxia (50 min) (29). In this later 
study, $10 \mathrm{U} / \mathrm{g}$ were injected but when the same total dose of Epo was administered at two times $(5 \mathrm{U} / \mathrm{g} 24 \mathrm{~h}$ and $4 \mathrm{~h}$ before $\mathrm{HI}$ ), the treatment was ineffective. This suggests that a high dose of Epo may be required to obtain a therapeutic effect in neonates. Kellert et al. (19) showed that the neuroprotective effect of Epo was dose-dependent and that the greatest effect was found with a $30 \mathrm{U} / \mathrm{g}$ dose after $\mathrm{HI}$ insult in $\mathrm{P} 7$ rats justifying our choice to use a unique dose of $30 \mathrm{U} / \mathrm{g}$ of Epo. However, even if high doses of Epo have been reported to be safe $(19,27)$, we acknowledge that this dosage may not be compatible with administration in humans.

Epo administration and FA in HI lesion. No difference was observed in FA values between the three groups. By measuring FA in the developing rat brain, Huang et al. (30) showed a progressive decrease in cortical areas in healthy neonatal rats at $0,3,7,11$, and $19 \mathrm{~d}$ of life, thus providing a standard allowing the detection of abnormalities in cortical development in neonatal animal models. Normal FA values measured in the cortical plate and mantle in healthy neonatal P7 rat brain in this study were 0.3-0.4. In a recent study on healthy rat neonates, Bockhorst et al. (31) found same values in these regions. This absence of difference might be due to the fact that MRI was performed very early after the insult. Our results are in accordance with this theory because $3 \mathrm{~h}$ after the carotid ligature, the mean FA values in the HI injury area in groups I, II, and III were similar and equal to 0.3, i.e., the value found by Huang et al. (30). A decrease in FA values did occur but later ( $24 \mathrm{~h}$ after the insult). A recent article by Wang et al. (32) explored white matter injury at D1 in an HI neonatal rat model by DTI and showed that DTI parameters represented by FA, trace, $\lambda$ parallel, and $\lambda$ perpendicular are useful in characterizing WM injury (in terms of severity of damage); $\lambda \mathrm{I}$ and $\lambda \perp$ in particular may make it possible to differentiate the axonal structure breakdown due to axonal swelling, axonal transport dysfunction, or irreversible histologic damage.

Epo administration and ADC measurement in stroke. The kinetics of ADC variations in neonatal $\mathrm{HI}$ rat is well understood (33-35). In injured cerebral cortex in the neonatal HI rat model, ADC values decrease early (from $1 \mathrm{~h}$ post-HI) with a statistical difference between lesion and contralateral hemisphere or control. In this later study, the same difference was observed $24 \mathrm{~h}$ post-HI. In a recent article, Wang explored the best ADC threshold in the injured hemisphere in comparison with the contralateral hemisphere to predict as best as possible the final size of HI injury in the neonatal HI rat model. They concluded that injured areas with an $80 \%$ ADC decrease compared with the contralateral hemisphere (1-2 h post-HI) should be considered to define the final $\mathrm{HI}$ injury size. A few days to a few weeks after injury, ADC values pseudonormalize in the lesion, indicating the destruction of tissue organization associated with an increased isotropy $(22,34)$. In our study, rats underwent DWI examination $3 \mathrm{~h}$ after HI injury. Our data concerning ADC values for $\mathrm{HI}$ rats are in accordance with the literature $(22,32)$ : the mean $\mathrm{ADC}$ value in $\mathrm{HI}$ injury was significantly lower than in the contralateral hemisphere (respectively $0.565 \times 10^{-3} \pm 0.086 \times 10^{-3}$ and $0.98 \times 10^{-3} \pm$ $0.08 \times 10^{-3} p>10^{-6}$ ). No difference exist between $\mathrm{ADC}$ value in the healthy hemisphere in groups II and III and ADC value in pup brain in group I.

Immunohistochemistry: caspase 3 and AQP4 expression in the neonatal brain. The lack of apoptosis markers (caspase 3) may be due to the early time point at which animals were killed after HI. In our study, Epo had an effect not only on brain surface lesions but also on ADC values. The mechanism of Epo on water diffusion remains uncertain. Because the control of water homeostasis is modulated by specific water channel proteins called aquaporin, we decided to look for the presence of AQP4 by immunohistochemistry. Aquaporin is a transmembrane protein (MW around 30,000 D) involved in the passage of water through the plasma membrane and across the blood brain barrier. AQP4 is mainly expressed in pericapillary astrocytic endfeet and is the major water channel in the brain $(18,36,37)$.

The expression of AQP4 thus increased in and around the damaged cortical area as soon as $3 \mathrm{~h}$ after carotid ligation in treated rats. Several studies have already shown the important function of AQP4 in respective cytotoxic and vasogenic brain edemas, glial cell migration, and neural signal transduction (38). A recent study by Tourdias et al. (39) hypothesized that up-regulation of AQP4 in brain, in response to hydrocephalus, may participate in clearance of excess fluid to the blood stream. That study was one of the first to correlate HI-induced changes in ADC in the periventricular edema to the spatial and temporal expression of AQP4. Interestingly, its authors observed that the higher the ADC values in this zone, the higher the AQP4 expression. Our results indicate a similar association between $\mathrm{ADC}$ in $\mathrm{HI}$ injury and $\mathrm{AQP} 4$ expression in the brain. Indeed, high-ADC values in the HI injury area in Epo-treated rat pups correspond to a high level of brain AQP4 expression.

It would be interesting to explore Epo receptor (Epo-R) expression in HI newborn rats treated by Epo to see whether Epo-R up-regulates in the $\mathrm{HI}$ hemisphere in correlation with increasing AQP4 expression. An increase in Epo and Epo-R mRNA has been reported in rodent and human brain after ischemia but with a different pattern (40). Epo-R may be rapidly up-regulated by $\mathrm{HI}$, whereas endogenous Epo protein expression increases only $24 \mathrm{~h}$ after, thus yielding a time lag in the response. Therefore, the administration of r-hu-Epo immediately after carotid ligature and just before hypoxia could compensate for this delay between Epo-R and endogenous Epo expression, leading to an early response and thus accounting for its neuroprotective action in the ischemic brain.

Limitations of the study. Body temperature control of the neonates during MRI was not possible (no amagnetic material), but the gradient system was heated by passing hot water inside to prevent the cooling of the animal. To limit bias, all the animals benefited from this care. Owing to technical limitations, it was not possible to explore more than one slice in our study. Thus, we could not explore the spatial FA and ADC distribution. Measurements for ADC and FA in the injured hemisphere were performed only in the HI injury area because it was not possible to create reproducible masks to compare these parameters in the caudate nucleus, for example. It would have been interesting to measure ADC and FA in 
regions without any apparent abnormality. Observers for DWI and immunohistochemistry were not masked to group assignment (Epo versus No Epo) AQP4 mRNA and Epo-R quantifications would also have been interesting in the understanding of Epo neuroprotective mechanisms.

\section{CONCLUSION}

We conclude that Epo has an early protective effect in HI, possibly through a rapid up-regulation of AQP4 expression. This effect seems to be immediate, in contrast to the classic "late" neuroprotective effects of Epo, that are known to depend on gene activation. One possible mechanism for the limited brain edema observed in the HI-Epo group could be a better clearance of water excess from brain tissues to blood by AQP4. The next step will be to perform DWI (ADC and FA measurements) and immunohistochemical (AQP-4) longitudinal studies on this neonatal HI rat model after Epo treatment to observe the possible long-term effect of this neuroprotective molecule.

Acknowledgments. We thank Janssen-Cilag who generously provided the r-hu-Epo and Dr. Thomas Tourdias for providing the histologic protocol for AQP4.

\section{REFERENCES}

1. Perlman JM 2006 Intervention strategies for neonatal hypoxic-ischemic cerebral injury. Clin Ther 28:1353-1365

2. Rice JE III, Vannucci RC, Brierley JB 1981 The influence of immaturity on hypoxic-ischemic brain damage in the rat. Ann Neurol 9:131-141

3. Hagberg H, Bona E, Gilland E, Puka-Sundvall M 1997 Hypoxia-ischaemia model in the 7-day-old rat: possibilities and shortcomings. Acta Paediatr Suppl 422:85-88

4. Sola A, Peng H, Rogido M, Wen TC 2008 Animal models of neonatal stroke and response to erythropoietin and cardiotrophin-1. Int J Dev Neurosci 26:27-35

5. Le Bihan D 2007 The 'wet mind': water and functional neuroimaging. Phys Med Biol 52:R57-R90

6. Ward P, Counsell S, Allsop J, Cowan F, Shen Y, Edwards D, Rutherford M 2006 Reduced fractional anisotropy on diffusion tensor magnetic resonance imaging after hypoxic-ischemic encephalopathy. Pediatrics 117:e619-e630

7. Buller KM, Carty ML, Reinebrant HE, Wixey JA 2009 Minocycline: a neuroprotective agent for hypoxic-ischemic brain injury in the neonate? J Neurosci Res 87:599-608

8. Chaudhari T, McGuire W 2008 Allopurinol for preventing mortality and morbidity in newborn infants with suspected hypoxic-ischaemic encephalopathy. Cochrane Database Syst Rev CD006817

9. van der Kooij MA, Groenendaal F, Kavelaars A, Heijnen CJ, van Bel F 2009 Combination of deferoxamine and erythropoietin: therapy for hypoxia-ischemiainduced brain injury in the neonatal rat? Neurosci Lett 451:109-113

10. Pazaiti A, Soubasi V, Spandou E, Karkavelas G, Georgiou T, Karalis P, GuibaTziampiri O 2009 Evaluation of long-lasting sensorimotor consequences following neonatal hypoxic-ischemic brain injury in rats: the neuroprotective role of $\mathrm{MgSO} 4$. Neonatology 95:33-40

11. Gonzalez FF, McQuillen P, Mu D, Chang Y, Wendland M, Vexler Z, Ferriero DM 2007 Erythropoietin enhances long-term neuroprotection and neurogenesis in neonatal stroke. Dev Neurosci 29:321-330

12. Kumral A, Ozer E, Yilmaz O, Akhisaroglu M, Gokmen N, Duman N, Ulukus C, Genc S, Ozkan H 2003 Neuroprotective effect of erythropoietin on hypoxic-ischemic brain injury in neonatal rats. Biol Neonate 83:224-228

13. Shannon KM, Keith JF III, Mentzer WC, Ehrenkranz RA, Brown MS, Widness JA, Gleason CA, Bifano EM, Millard DD, Davis CB, Stevenson DK, Alverson DC, Simmons CF, Brim M, Abels RI, Phibbs RH 1995 Recombinant human erythropoietin stimulates erythropoiesis and reduces erythrocyte transfusions in very low birth weight preterm infants. Pediatrics 95:1-8

14. Rabie T, Marti HH 2008 Brain protection by erythropoietin: a manifold task. Physiology (Bethesda) 23:263-274
15. Sola A, Rogido M, Lee BH, Genetta T, Wen TC 2005 Erythropoietin after focal cerebral ischemia activates the Janus kinase-signal transducer and activator of transcription signaling pathway and improves brain injury in postnatal day 7 rats. Pediatr Res 57:481-487

16. Verdonck O, Lahrech H, Francony G, Carle O, Farion R, Van de Looij Y, Remy C, Segebarth C, Payen JF 2007 Erythropoietin protects from post-traumatic edema in the rat brain. J Cereb Blood Flow Metab 27:1369-1376

17. Chang YS, Mu D, Wendland M, Sheldon RA, Vexler ZS, McQuillen PS, Ferriero DM 2005 Erythropoietin improves functional and histological outcome in neonatal stroke. Pediatr Res 58:106-111

18. Gunnarson E, Song Y, Kowalewski JM, Brismar H, Brines M, Cerami A, Andersson U, Zelenina M, Aperia A 2009 Erythropoietin modulation of astrocyte water permeability as a component of neuroprotection. Proc Natl Acad Sci USA 106:1602-1607

19. Kellert BA, McPherson RJ, Juul SE 2007 A comparison of high-dose recombinant erythropoietin treatment regimens in brain-injured neonatal rats. Pediatr Res 61:451-455

20. Ashwal S, Tone B, Tian HR, Chong S, Obenaus A 2007 Comparison of two neonatal ischemic injury models using magnetic resonance imaging. Pediatr Res 61:9-14

21. Yang J, Wu EX 2007 Manganese-enhanced MRI detected the gray matter lesions in the late phase of mild hypoxic-ischemic injury in neonatal rat. Conf Proc IEEE Eng Med Biol Soc:2007;51-54

22. van der Zijden JP, van der Toorn A, van der Marel K, Dijkhuizen RM 2008 Longitudinal in vivo MRI of alterations in perilesional tissue after transient ischemic stroke in rats. Exp Neurol 212:207-212

23. Fau S, Po C, Gillet B, Sizonenko S, Mariani J, Meric P, Charriaut-Marlangue C 2007 Effect of the reperfusion after cerebral ischemia in neonatal rats using MRI monitoring. Exp Neurol 208:297-304

24. Rumpel H, Buchli R, Gehrmann J, Aguzzi A, Illi O, Martin E 1995 Magnetic resonance imaging of brain edema in the neonatal rat: a comparison of short and long term hypoxia-ischemia. Pediatr Res 38:113-118

25. Wen TC, Rogido M, Peng H, Genetta T, Moore J, Sola A 2006 Gender differences in long-term beneficial effects of erythropoietin given after neonatal stroke in postnatal day-7 rats. Neuroscience 139:803-811

26. Demers EJ, McPherson RJ, Juul SE 2005 Erythropoietin protects dopaminergic neurons and improves neurobehavioral outcomes in juvenile rats after neonatal hypoxia-ischemia. Pediatr Res 58:297-301

27. Statler PA, McPherson RJ, Bauer LA, Kellert BA, Juul SE 2007 Pharmacokinetics of high-dose recombinant erythropoietin in plasma and brain of neonatal rats. Pediatr Res 61:671-675

28. van der Kooij MA, Groenendaal F, Kavelaars A, Heijnen CJ, van Bel F 2008 Neuroprotective properties and mechanisms of erythropoietin in in vitro and in vivo experimental models for hypoxia/ischemia. Brain Res Rev 59:22-33

29. Wang X, Zhu C, Gerwien JG, Schrattenholz A, Sandberg M, Leist M, Blomgren K 2004 The nonerythropoietic asialoerythropoietin protects against neonatal hypoxiaischemia as potently as erythropoietin. J Neurochem 91:900-910

30. Huang H, Yamamoto A, Hossain MA, Younes L, Mori S 2008 Quantitative cortical mapping of fractional anisotropy in developing rat brains. J Neurosci 28:1427-1433

31. Bockhorst KH, Narayana PA, Liu R, Ahobila-Vijjula P, Ramu J, Kamel M, Wosik J, Bockhorst T, Hahn K, Hasan KM, Perez-Polo JR 2008 Early postnatal development of rat brain: in vivo diffusion tensor imaging. J Neurosci Res 86:1520-1528

32. Wang S, Wu EX, Tam CN, Lau HF, Cheung PT, Khong PL 2008 Characterization of white matter injury in a hypoxic-ischemic neonatal rat model by diffusion tensor MRI. Stroke 39:2348-2353

33. Meng S, Qiao M, Foniok T, Tuor UI 2005 White matter damage precedes that in gray matter despite similar magnetic resonance imaging changes following cerebral hypoxia-ischemia in neonatal rats. Exp Brain Res 166:56-60

34. Meng S, Qiao M, Scobie K, Tomanek B, Tuor UI 2006 Evolution of magnetic resonance imaging changes associated with cerebral hypoxia-ischemia and a relatively selective white matter injury in neonatal rats. Pediatr Res 59:554-559

35. Qiao M, Meng S, Scobie K, Foniok T, Tuor UI 2004 Magnetic resonance imaging of differential gray versus white matter injury following a mild or moderate hypoxic-ischemic insult in neonatal rats. Neurosci Lett 368:332-336

36. Badaut J, Lasbennes F, Magistretti PJ, Regli L 2002 Aquaporins in brain: distribution, physiology, and pathophysiology. J Cereb Blood Flow Metab 22:367-378

37. Hirt L, Ternon B, Price M, Mastour N, Brunet JF, Badaut J 2009 Protective role of early aquaporin 4 induction against postischemic edema formation. J Cereb Blood Flow Metab 29:423-433

38. Verkman AS, Binder DK, Bloch O, Auguste K, Papadopoulos MC 2006 Three distinct roles of aquaporin- 4 in brain function revealed by knockout mice. Biochim Biophys Acta 1758:1085-1093

39. Tourdias T, Dragonu I, Fushimi Y, Deloire MS, Boiziau C, Brochet B, Moonen C, Petry KG, Dousset V 2009 Aquaporin 4 correlates with apparent diffusion coefficient and hydrocephalus severity in the rat brain: a combined MRI-histological study. Neuroimage 47:659-666

40. Spandou E, Papoutsopoulou S, Soubasi V, Karkavelas G, Simeonidou C, Kremenopoulos G, Guiba-Tziampiri O 2004 Hypoxia-ischemia affects erythropoietin and erythropoietin receptor expression pattern in the neonatal rat brain. Brain Res 1021:167-172 